\title{
Level-1 Muon Triggers for the CMS Experiment at the HL-LHC
}

\section{Santiago Folgueras ${ }^{* \dagger}$}

Universidad de Oviedo, Instituto Universitario de Ciencias y Tecnologías Espaciales de Asturias (ICTEA), Spain

E-mail: folguerassantiago@uniovi.es

The High Luminosity upgrade of the CERN LHC will deliver proton-proton collisions at $14 \mathrm{TeV}$ with instantaneous luminosities up to $7.5 \times 10^{34} \mathrm{~cm}^{-2} \mathrm{~s}-1$. The physics program of the Compact Muon Solenoid experiment strongly depends on the ability to identify muons at an early trigger stage, over a momentum range spanning from few $\mathrm{GeV}$ to the $\mathrm{TeV}$ scale. Several upgrades of the muon triggers are foreseen in order to retain such capabilities in view of the the High Luminosity LHC, including the acceptance on electroweak processes and the sensitivity to physics beyond the Standard Model. With this contribution, we review the current status of the design of highly efficient muon trigger, its architecture, based on state-of-the-art Field Programmable Gate Array processors and O(10) Gbps serial optical links, and the foreseen muon identification algorithms. We discuss the local trigger primitives generation, depending on the new read-out of several detectors, their increased acceptance, and on-line muon reconstruction algorithms, both standalone and with the contribution of the silicon tracker at Level-1. The expected benefits for the High Luminosity LHC physics program are presented as well.

European Physical Society Conference on High Energy Physics - EPS-HEP2019 -

10-17 July, 2019

Ghent, Belgium

\author{
* Speaker. \\ ${ }^{\dagger}$ On behalf of the CMS Collaboration
}




\section{Introduction}

The High Luminosity Large Hadron Collider (HL-LHC) will open up an incredible opportunity for high energy physics: the expected collected data set the collected data set will be 10-20 times larger than the one we have today and it will facilitate to perform both high-precision Standard Model (SM) measurements, such as, the properties of the Higgs boson; as well as access to unconventional signatures and corners of the phase space which may allow for a discovery.

The trigger system of the Compact Muon Solenoid (CMS) experiment comprises two levels [1]. The L1 trigger consists of custom hardware processors that receive data from calorimeter and muon systems, generating a trigger signal within $3.8 \mu \mathrm{s}$, with a maximum rate of $100 \mathrm{kHz}$. The full detector is read out on receipt of a Level-1 Accept (L1A) signal, and events are built. The HighLevel Trigger (HLT) is implemented in software and reduces the rate to $1 \mathrm{kHz}$. This two-level strategy will not change for HL-LHC (Phase-2 upgrades), although the entire trigger and the data acquisition system will be replaced. The detector readout electronics will be upgraded to allow a maximum L1A rate of $750 \mathrm{kHz}$, and a latency of $12.5 \mu \mathrm{s}$. The upgraded system will be entirely running on commercial Field Programmable Gate Array (FPGA) processors and should greatly extend the capabilities of the current system, being able to maintain trigger thresholds despite the harsh environment as well as triggering on more exotic signatures such as long-lived particles (LLPs), or Heavy Stable Charged Particles (HSCP) to extend the physics coverage.

Muons are detected with multi-layer gas-ionization detectors positioned outside the solenoid, highlighted in Fig. 1. Three different sub-detectors can be distinguished: Drift Tube chambers (DTs) in the barrel region (extending up to $|\eta|<1.2$ ), Cathode Strip Chambers (CSCs) in the endcap region (extending from $0.9<|\eta|<2.4$ ) and Resistive Plate Chambers (RPCs) reaching up to $|\eta|<1.9$. There are four muon stations, both in the barrel and in each endcap. The upgraded muon system will extend the RPC coverage up to $|\eta|<2.5$ with the improved RPC (iRPC), and add a fourth sub-detector, Gas Electron Multipliers (GEMs) which will increase the redundancy of the system in the region $1.6<|\eta|<2.5$. Three distinct geometrical regions can also be identified in Fig. 1, referred to as: barrel $(|\eta|<0.9)$, overlap $(0.9<|\eta|<1.2)$, and endcap $(|\eta|>1.2)$. A more detailed description can be found elsewhere [2].

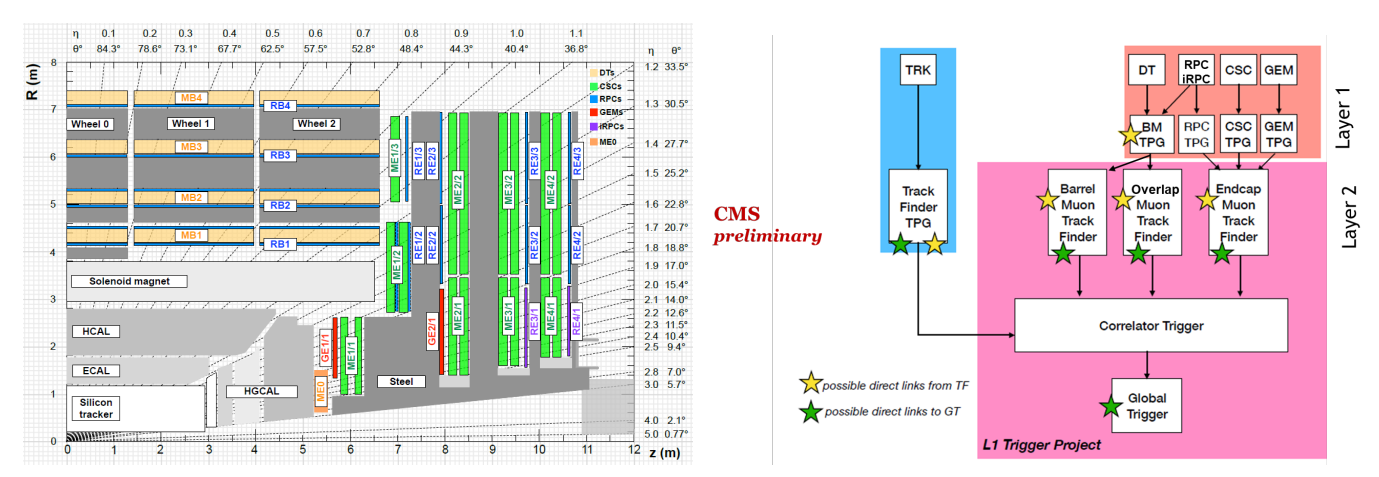

Figure 1: Left: The upgraded muon system, the different muon sub-detectors are highlighted. Right: The Phase-2 L1 muon trigger architecture (the picture only shows those elements relevant to the muon trigger). From [1][2]. 
The proposed Phase-2 upgrade architecture of the L1 Muon Trigger system is staged in a two-layer system: the first layer collects the input from different sub-detectors, to generate locallyreconstructed segments called trigger primitives (TPs). Then the muon track finders, collect the different TPs and run pattern algorithms and estimate the momentum. The two layers are closely interconnected, and the performance of the second will strongly depend on the first layer. In the following pages a brief description of the algorithms that are being designed for each layer is given. Details on the proposed architecture and algorithms can be found in Ref. [1].

\section{Local muon reconstruction (trigger primitives)}

\subsection{Trigger primitive generation in the barrel}

The current barrel muon trigger primitives consist of local muon stubs from the DT chambers, and hits from the RPC system. The goals for the Phase-2 trigger primitive generation include maximising efficiency, exploiting the full spatial resolution of the DT system, and improving the time resolution of RPC clusters delivered to the trigger from $25 \mathrm{~ns}$ to $1 \mathrm{~ns}$. The design algorithm must be robust against potential ageing of the muon detector. The trigger primitive generation will be performed by an asynchronous algorithm implemented on a commercial FPGA processor. This approach opens the possibility to produce local trigger primitives, with only the intrinsic limitations of the DT chamber, since all chamber hits and their highest resolution time stamps will be forwarded to the off-detector electronics. Two different algorithms are being considered:

Analytical Method Used for trigger primitive generation aims at exploiting the full resolution that can be achieved after the DT Phase 2 upgrade. It performs a muon trajectory reconstruction algorithm based on the mean-timer paradigm. The inputs of this algorithm are the TDC time and cell number of all signals detected in a superlayer (SL) and assumes that muons follow a straight path inside a each chamber. This algorithm selects patterns using at least 3 fired cells for each SL independently, and estimates the time and slope of the muon trajectory using the mean-timer equations. As a final step, the information of the two SL is correlated if two muon stubs are found in the same bunch-crossing. Figure 2 (left) shows the slope resolution obtained after correlating stubs from two SLs. A first version of this algorithm has been already taking data in the slice-test using prototypes for the Phase-2 electronics. Recent developments explore the possibility of using the two SLs at once which will increase the robustness against potential ageing effects.

Histogram-Based A second approach has also being tested using a histogram-based mean-timer technique for the bunch crossing identification, plus a chamber level track segment fitting based on a Compact Hough Transform. This method already looks at the information of the entire DT chamber at once, improving time and slope resolution of the TPs from one-single SL.

\subsection{Trigger primitive generation in the endcap}

The endcap muon system currently comprises CSC and RPC detectors. By the time of the HL-LHC, the coverage will have been extended by the addition of iRPC and GEM chambers. All detectors will provide TPs to the L1 trigger. The TPs for existing detectors will not change, except 

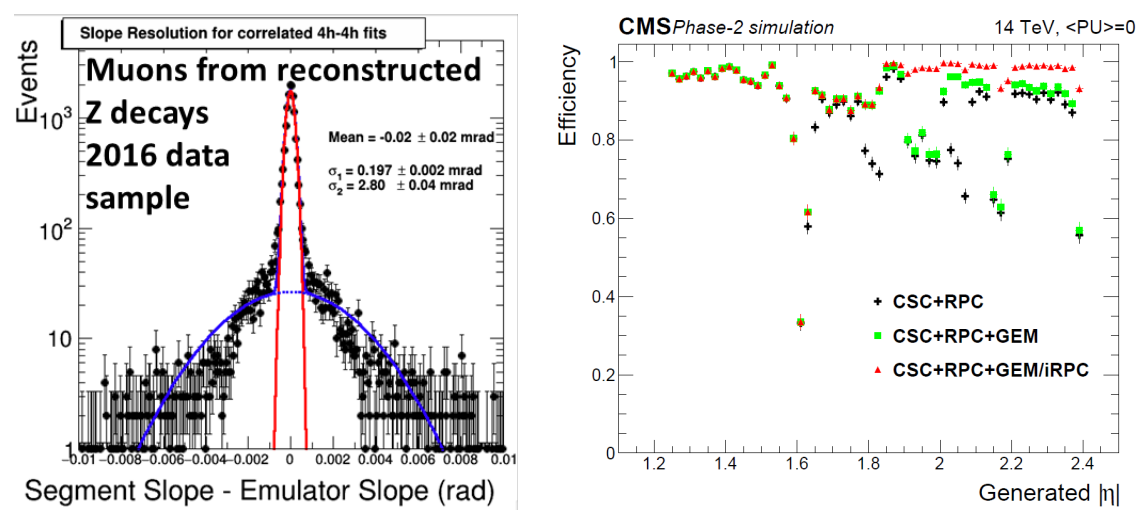

Figure 2: Performance of the Phase-2 upgrade trigger primitive generation. Left shows the slope resolution of the barrel algorithm. Right displays the efficiency for the endcap. From [2].

when combining local information across detectors. Improvements to the stub reconstruction algorithm are envisaged to mitigate inefficiencies that arise at high pileup. These include improved ghost (i.e. ambiguous and/or fake) track cancellation logic, reduction of pre-trigger dead-time, optimised pattern recognition, and improved timing. The efficiency improvement is visible in Fig. 2 (right).

\section{Online muon reconstruction (track finders)}

The HL upgrade will significantly extend the capabilities of Run 2 muon track finders (MTF), in addition to the standard (standalone) muon reconstruction, the MTF will be able to run dedicated reconstruction algorithms aiming at triggering on muons that do not point to the primary vertex, and HSCPs. Furthermore, tracking information will also be accessible and thus correlating muon and tracker information could be done before the L1A decision is made. Due to the different subdetector technologies, different geometries and magnetic field, each of the regions of the muon detector (barrel, overlap and endcap) provide different solutions to consider the multiple-scattering and the propagation of the muon from chamber to chamber through the magnetic field.

\subsection{Barrel muon track finder (BMTF): Kalman filter}

The barrel muon trigger architecture is designed with the goal of reducing latency and increasing performance with respect to the Phase-1 muon trigger. The proposed algorithm utilizes an iterative track finding approach that combines information from different muon stubs in every station. The chamber-to-chamber propagation is based on a Kalman filter to determine the muon momentum. The iterative approach starts from the outermost muon station, propagates inward and updates at each station. It provides two measurement of the momentum: one vertex constrained and one unconstrained. A preliminary version has been already implemented in firmware, with small logic occupancy $(<20 \%)$ and a total latency of 10 bunch-crossings, and has been already used at the end of 2018 data taking as a main trigger algorithm. The improvement with respect to the Phase-1 algorithm is depicted in Fig. 3. 

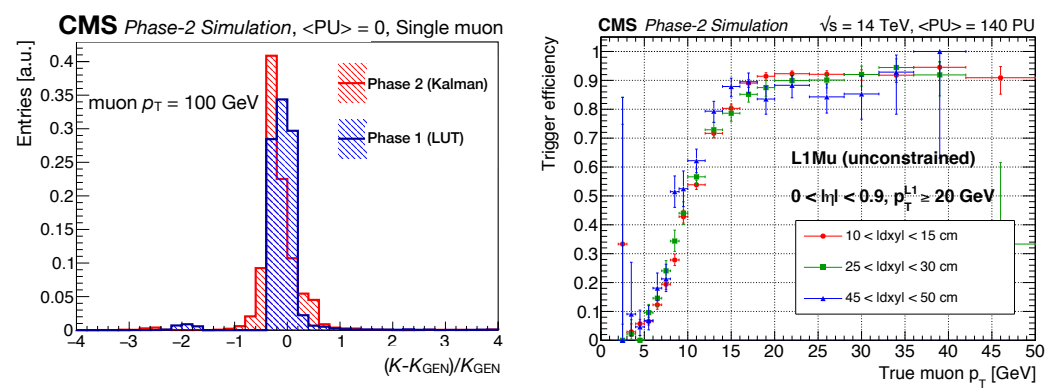

Figure 3: Left: Barrel muon track finder resolution improvement with respect to the Phase-1 implementation for $100 \mathrm{GeV}$ muons. Efficiencies of the displaced muon algorithm in the barrel for impact parameters between 10-15 cm (solid red circles), 25-30 cm (solid green squares), and 45-50 cm (solid blue triangles). From [1].

Future searches at the Run 3 should cover a large phase space and, ideally, should not be driven by strict model assumptions. Many new-physics scenarios predict the existence of LLPs that may decay into muons that are significantly displaced from the beam line (displaced muons). Triggering on those muons, however, is extremely challenging as they will be reconstructed as low momentum muons that cannot be accepted by the L1A decision. The unique capabilities of the muon system allow to trigger on those muons by simply removing the vertex constrain from the track fitting (see Fig.3 (right)). A customized displaced Kalman-BMTF has been designed and improves efficiency by a factor of 6 for highly displaced muons. As a momentum estimate it considers the maximum between the constrained and unconstrained $p_{\mathrm{T}}$ assignment.

\subsection{Overlap muon track finder (OMTF): Naïve-Bayes Classifier}

The pseudorapitidy region where barrel and endcaps overlap, has a particularly difficult geometry with detector layers oriented both parallel and perpendicular to the beam line. Moreover it is a region with an especially non-homogeneous magnetic field. The particularities of this region requires a dedicated MTF partition and a special algorithm dedicated to this region. The algorithm combines information from 18 separate detector layers: 6 from DT (3 layers with position and 3 bending measurements), 5 RPC barrel layers, 3 RPC endcap layers, and 4 layers from CSC. The algorithm implements a Naïve-Bayes Classifier for muon identification and momentum measurement in one step by minimizing a Likelihood. It assumes that the log-likelihood that a muon has a given $p_{\mathrm{T}}$ hypothesis is just the sum of the likelihood of a muon hit $\phi$ position in each layer. The maximum $\log$-likelihood $p_{\mathrm{T}}$ is chosen as the muon momentum. This algorithm has already being used in Run 2 data-taking and a simple extension works for HL-LHC. The turn-on curve as a function of $p_{\mathrm{T}}$ for the OMTF algorithm in 2018 data is shown in Fig. 4.

\subsection{Endcap muon track finder (EMFT): Neural Networks}

The architecture of the Phase-2 EMTF is similar to the present Phase-1 trigger, however new muon detectors will need to be incorporated that will improve efficiency, timing and momentum assignment. Redundancy in the full pseudo-rapidity region will help with local efficiency reconstruction, $p_{\mathrm{T}}$ resolution, rate reduction and resilience to ageing effects. The expected performance 


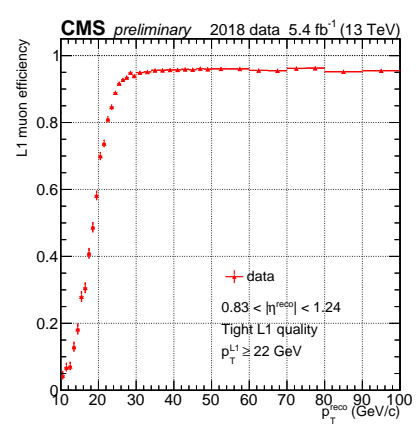

Figure 4: Efficiency of the OMTF as a function of the $p_{\mathrm{T}}$ for 2018 data. For the HL-LHC we expect to at lease maintain the performance achieved so far while improving momentum resolution. Triggering on unconventional signatures will also be feasible [3].

in the HL-LHC scenario is depicted in Fig. 5: a sharper turn-on curve than that of the Phase-1 EMTF and higher efficiency thanks to the new sub-detectors is shown. The rate is also reduced.

Another improvement arises from the Phase-2 algorithm that is being designed: a NeuralNetwork-based for track building and momentum assignment, following same design as current EMTF. New track patterns are being trained to take advantage of the additional stations. The output of the network assigns a momentum to each muon, and provides an estimate on the pile-up, which greatly reduces the rate.
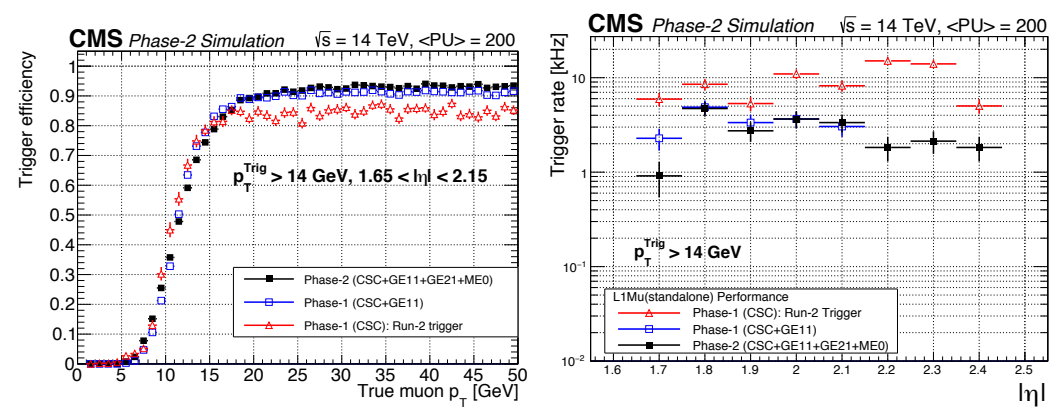

Figure 5: EMTF expected performance improvement. Left shows the efficiency as a function of the muon $p_{\mathrm{T}}$. Right shows the expected trigger rate for a given momentum threshold as a function of the pseudorapidity. From [1].

\subsection{Track-muon correlator}

The availability of tracking information at L1 is likely the greatest change in paradigm in the L1 trigger. Momentum assignment will be extremely precise in the tracker. A track-muon correlator is therefore needed to match track-trigger tracks and muon tracks, following an approach very similar to that used offline: Either track-trigger tracks are matched with single muon stubs to improve efficiency, or muon tracks are matched with track-trigger tracks to improve the momentum assignment from the MTFs. Different solutions based on the algorithms from the muon track finders are being considered for this track-muon matching, providing similar performances. 
The access to tracker tracks allows for many new features among which is triggering on HSCPs. Such particles may appear in many new physics models and may never be detected unless a special trigger is designed. The HSCP will cross the entire detectors moving slowly, so they will look like muons, only with much smaller speed $(\beta<0.8)$. In the most simple case the particle will be produced at the primary vertex and will look like a high-momentum track, the task is to tag it as a slow particle. Two options have been considered: either use RPC-only hits to determine speed, or, as tracks from $15 \mathrm{BX}$ will be sent to the muon correlator, match tracks to muon stubs in 3 different BXs using a Naïve-Bayes Classifier. The upgraded algorithms show greater resolution and efficiency for slow-moving particles (see Fig. 6).
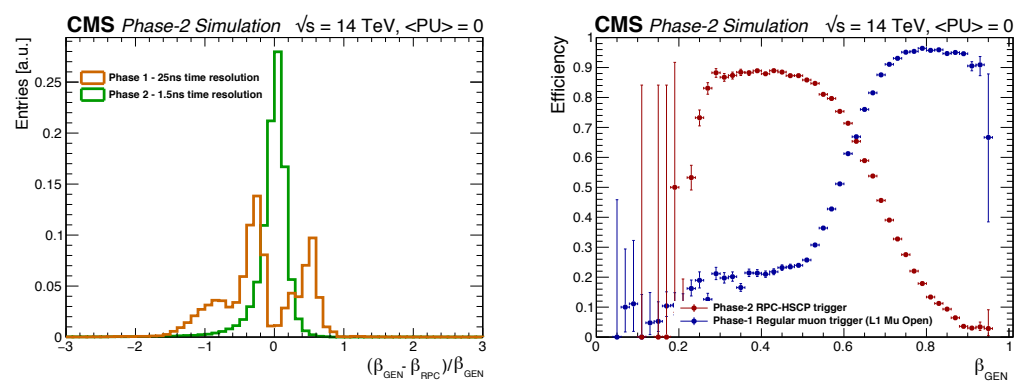

Figure 6: Resolution of the $\beta$ measurement for L1 muon tracks using L1 trigger RPC hits (left), and efficiency for identifying HSCPs as a function of $\beta$ for Phase-1 and Phase-2 L1 muon triggers (right). From [1].

\section{Conclusions}

The High Luminosity LHC upgrades will significantly extend the capabilities of the Level1 muon triggers. First, they will allow for a higher bandwidth and latency. Second, algorithm improvements will allow not to increase the current trigger thresholds despite the harsher environment. Third, triggering on unique signatures such as displaced muons or Heavy Stable Charged Particles. The expected improvements will already be visible at the Level-1 trigger primitive generation thanks to the use of Field Programmable Gate Arrays and the combination of information from various sub-detectors. In addition, the muon track finders will greatly improve momentum resolution with respect to that of the current detector, thanks to the use of the L1 tracker tracks. A Technical Design Report with greater details on the proposed algorithms and designed architecture is expected in 2020 .

\section{References}

[1] CMS Collaboration, "The Phase-2 Upgrade of the CMS L1 Trigger Interim Technical Design Report”, CERN-LHCC-2017-013. CMS-TDR-017.

[2] CMS Collaboration, "The Phase-2 Upgrade of the CMS Muon Detectors”, CERN-LHCC-2017-012. CMS-TDR-016.

[3] CMS Collaboration, "Level-1 Muon Trigger Performance”, CERN-CMS-DP-2018-044. CMS-DP-2018-044 